June 4, 2010

\title{
A SHARP BOUND ON EIGENVALUES OF SCHRÖDINGER OPERATORS ON THE HALFLINE WITH COMPLEX-VALUED POTENTIALS
}

\author{
RUPERT L. FRANK, ARI LAPTEV, AND ROBERT SEIRINGER
}

Abstract. We derive a sharp bound on the location of non-positive eigenvalues of Schrödinger operators on the halfline with complex-valued potentials.

\section{IntRodUCTION AND MAIN RESULT}

In this note we are concerned with estimates for non-positive eigenvalues of one-dimensional Schrödinger operators with complex-valued potentials. We shall provide an example of a bound where the sharp constant worsens when a Dirichlet boundary condition is imposed. This is in contrast to the case of real-valued potentials, where the variational principle implies that the absolute value of the non-positive eigenvalues decreases.

In order to describe our result, we first assume that $V$ is real-valued. It is a well-known fact (attributed to L. Spruch in $[\mathrm{K}]$ ) that any negative eigenvalue $\lambda$ of the Schrödinger operator $-\partial^{2}-V$ in $L^{2}(\mathbb{R})$ satisfies

$$
|\lambda|^{1 / 2} \leq \frac{1}{2} \int_{-\infty}^{\infty}|V(x)| \mathrm{d} x .
$$

The constant $\frac{1}{2}$ in this inequality is sharp and attained if $V(x)=c \delta(x-b)$ for any $c>0$ and $b \in \mathbb{R}$. (It follows from the Sobolev embedding theorem that the operator $-\partial^{2}-V$ can be defined in the quadratic form sense as long as $V$ is a finite Borel measure on $\mathbb{R}$. In this case the right side of (11) denotes the total variation of the measure.) From (1) and the variational principle for self-adjoint operators we immediately infer that any negative eigenvalue of the operator $-\partial^{2}-V$ in $L^{2}(0, \infty)$ with Dirichlet boundary conditions satisfies

$$
|\lambda|^{1 / 2} \leq \frac{1}{2} \int_{0}^{\infty}|V(x)| \mathrm{d} x .
$$

The constant $\frac{1}{2}$ in this inequality is still sharp but no longer attained.

Motivated by concrete physical examples and problems in computational mathematics, an increasing interest in eigenvalue estimates for complex-valued potentials has developed in recent years. A beautiful observation of $\mathrm{AAD}$ is that (1) remains valid for all eigenvalues in $\mathbb{C} \backslash[0, \infty)$ even if $V$ is complex-valued. The same is not true for (2)! Indeed, our main result is

(C) 2009 by the authors. This paper may be reproduced, in its entirety, for non-commercial purposes.

Support through DFG grant FR 2664/1-1 (R.F.) and U.S. National Science Foundation grants PHY 0652854 (R.F.) and PHY 0652356 (R.S.) is gratefully acknowledged. 
Theorem 1.1. For $a \in \mathbb{R}$ let

$$
g(a):=\sup _{y \geq 0}\left|\mathrm{e}^{i a y}-\mathrm{e}^{-y}\right| .
$$

Any eigenvalue $\lambda=|\lambda| e^{i \theta} \in \mathbb{C} \backslash[0, \infty)$ of the operator $-\partial^{2}-V$ in $L^{2}(0, \infty)$ with Dirichlet boundary conditions satisfies

$$
|\lambda|^{1 / 2} \leq \frac{1}{2} g(\cot (\theta / 2)) \int_{0}^{\infty}|V(x)| \mathrm{d} x .
$$

This bound is sharp in the following sense: For any given $m>0$ and $\theta \in(0,2 \pi)$ there are $c \in \mathbb{C}$ and $b>0$ such that for $V(x)=c \delta(x-b)$ one has $|c|=\int|V(x)| \mathrm{d} x=$ $m$ and the unique eigenvalue of $-\partial^{2}-V$ is given by $\left(m^{2} / 4\right) g(\cot (\theta / 2))^{2} e^{i \theta}$, that is, equality is attained in (4).

Remark 1.2. Our bound does not apply to positive eigenvalues. In the case of realvalued potential it is known that there are no positive eigenvalues if $V \in L^{1}(\mathbb{R})$.

We note that $1<g(a)<2$ for $a>0$. The following lemma discusses the function $g$ in more detail.

Lemma 1.3. For $a \geq 0$, the function $g(a)$ is monotone increasing, with $g(0)=1$ and $\lim _{a \rightarrow \infty} g(a)=2$. Moreover,

$$
g(a)=1+O\left(\mathrm{e}^{-\pi /(3 a)}\right)
$$

for small a, and

as $a \rightarrow \infty$.

$$
g(a)=2-\frac{\pi}{a}+O\left(a^{-2}\right)
$$

In Figure 1 we plot the curve $\left\{|z|=g(\cot (\theta / 2))^{2}\right\}$. It follows from (6) that this curve hits the positive real axis at the point 4 with slope $2 / \pi$. Close to the point -1 the curve coincides with a semi-circle up to exponentially small terms, as (5) shows.

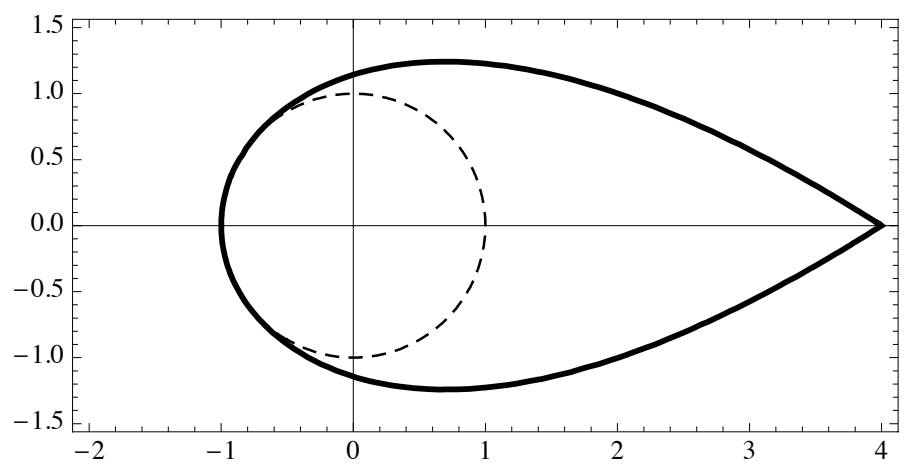

Figure 1. The maximal value of $4|\lambda|$ on the half-line with $\int_{0}^{\infty}|V(x)| \mathrm{d} x=1$. The dashed line is the corresponding bound on the whole line.

Using that $\sup _{a} g(a)=2$ we find 
Corollary 1.4. Any eigenvalue $\lambda \in \mathbb{C} \backslash[0, \infty)$ of the operator $-\partial^{2}-V$ in $L^{2}(0, \infty)$ with Dirichlet boundary conditions satisfies

$$
|\lambda|^{1 / 2} \leq \int_{0}^{\infty}|V(x)| \mathrm{d} x .
$$

The bound is not true in general if the right side is multiplied by a constant $<1$.

Inequality (7) follows also from inequality (11) for complex-valued potentials. Indeed, the odd extension of an eigenfunction of the Dirichlet operator is an eigenfunction of the whole-line operator with the potential $V(|x|)$ with the same eigenvalue. The remarkable fact is that the inequality is sharp in the complex-valued case, as shown in Theorem 1.1

By the same argument (7) is also valid if Neumann instead of Dirichlet boundary conditions are imposed. In this case equality holds for any $V(x)=c \delta(x)$ with $\operatorname{Re} c>0$. In particular, in the Neumann case (7) is sharp for any fixed argument $0<\theta<2 \pi$ of the eigenvalue $\lambda$. The analogue for mixed boundary conditions is

Proposition 1.5. Let $\sigma \geq 0$. Any eigenvalue $\lambda \in \mathbb{C} \backslash[0, \infty)$ of the operator $-\partial^{2}-V$ in $L^{2}(0, \infty)$ with boundary conditions $\psi^{\prime}(0)=\sigma \psi(0)$ satisfies

$$
|\lambda|^{1 / 2} \leq \int_{0}^{\infty}|V(x)| \mathrm{d} x .
$$

The bound is sharp for any $\sigma \geq 0$ and any fixed argument $0<\theta<2 \pi$ of the eigenvalue $\lambda$.

Note that if $\sigma<0$ a bound of the form (8) can not hold since there exists a non-positive eigenvalue even in the case $V=0$.

Remark 1.6. In the self-adjoint case inequality (11) for whole-line operators is accompanied by bounds

$$
|\lambda|^{\gamma} \leq \frac{\Gamma(\gamma+1)}{\sqrt{\pi} \Gamma(\gamma+3 / 2)}\left(\frac{\gamma-1 / 2}{\gamma+1 / 2}\right)^{\gamma-1 / 2} \int_{-\infty}^{\infty}|V(x)|^{\gamma+1 / 2} \mathrm{~d} x
$$

for $\gamma>1 / 2$; see $[\mathrm{K}, \mathrm{LT}$. In contrast, in the non-selfadjoint case it seems to be unknown whether the condition $V \in L^{\gamma+1 / 2}(\mathbb{R})$ for some $1 / 2<\gamma<\infty$ implies that all eigenvalues in $\mathbb{C} \backslash[0, \infty)$ lie inside a finite disc; see [DN, FLLS, LS, $\underline{\text { S] }}$ for partial results in this direction. We would like to remark here that even if a bound of the form (9) were true in the non-selfadjoint case with $1 / 2<\gamma<\infty$, then (in contrast to (11) for $\gamma=1 / 2$ ) the constant would have to be strictly larger than in the self-adjoint case. To see this, consider $V(x)=\frac{\alpha(\alpha+1)}{\cosh ^{2} x}$ with $\operatorname{Re} \alpha>0$. Then $\lambda=-\alpha^{2}$ is an eigenvalue (with eigenfunction $\left.(\cosh x)^{-\alpha}\right)$ and the supremum

$$
\sup _{\operatorname{Re} \alpha \geq 0} \frac{|\lambda|^{\gamma}}{\int_{-\infty}^{\infty}|V(x)|^{\gamma+1 / 2} \mathrm{~d} x}=\left(\int_{-\infty}^{\infty} \frac{d x}{\cosh ^{2} x}\right)^{-1} \sup _{\operatorname{Re} \alpha \geq 0} \frac{|\alpha|^{\gamma-1 / 2}}{|\alpha+1|^{\gamma+1 / 2}}
$$

is clearly attained for purely imaginary values of $\alpha$.

\section{Proofs}

Proof of Theorem 1.1. Assume that $-\partial^{2} \psi(x)-V(x) \psi(x)=-\mu \psi(x)$ with $\psi(0)=0$, $\psi \not \equiv 0$ and $\mu=-\lambda \in \mathbb{C} \backslash(-\infty, 0]$. Then the Birman-Schwinger operator

$$
V^{1 / 2} \frac{1}{-\partial^{2}+\mu}|V|^{1 / 2}, \quad V^{1 / 2}:=(\operatorname{sgn} V)|V|^{1 / 2},
$$


has an eigenvalue 1, and hence its operator norm is greater or equal to 1.

The integral kernel of this operator equals

$$
V(x)^{1 / 2} \frac{\mathrm{e}^{-\sqrt{\mu}|x-y|}-\mathrm{e}^{-\sqrt{\mu}(x+y)}}{2 \sqrt{\mu}}|V(y)|^{1 / 2},
$$

and hence

$$
\left|\left(\psi, V^{1 / 2} \frac{1}{-\partial^{2}+\mu}|V|^{1 / 2} \varphi\right)\right| \leq \frac{\|V\|_{1}}{2 \sqrt{|\mu|}}\|\psi\|_{2}\|\varphi\|_{2} \sup _{x, y \geq 0}\left|\mathrm{e}^{-\sqrt{\mu}|x-y|}-\mathrm{e}^{-\sqrt{\mu}(x+y)}\right| .
$$

Without loss of generality, we can take the supremum over the smaller set $x \geq y \geq 0$. Then

$$
\sup _{x \geq y \geq 0}\left|\mathrm{e}^{-\sqrt{\mu}(x-y)}-\mathrm{e}^{-\sqrt{\mu}(x+y)}\right|=\sup _{x \geq y \geq 0} \mathrm{e}^{-x \operatorname{Re} \sqrt{\mu}}\left|\mathrm{e}^{\sqrt{\mu} y}-\mathrm{e}^{-\sqrt{\mu} y}\right| .
$$

Since $\operatorname{Re} \sqrt{\mu}>0$, the supremum over $x$ is achieved at $x=y$, and hence

$$
\sup _{x, y \geq 0}\left|\mathrm{e}^{-\sqrt{\mu}(x-y)}-\mathrm{e}^{-\sqrt{\mu}(x+y)}\right|=\sup _{y \geq 0}\left|1-\mathrm{e}^{-2 \sqrt{\mu} y}\right| \text {. }
$$

If we write $\mu=-|\mu| \mathrm{e}^{i \theta}$ with $0<\theta<2 \pi$, then

$$
\sup _{y \geq 0}\left|1-\mathrm{e}^{-2 \sqrt{\mu} y}\right|=\sup _{y \geq 0}\left|\mathrm{e}^{2 i \sqrt{|\mu|} \cos (\theta / 2) y}-\mathrm{e}^{-2 \sqrt{|\mu|} \sin (\theta / 2) y}\right|=g(\cot (\theta / 2))
$$

with $g$ from (3). Hence we have shown that

$$
\left\|V^{1 / 2} \frac{1}{-\partial^{2}+\mu}|V|^{1 / 2}\right\| \leq \frac{\|V\|_{1}}{2 \sqrt{|\mu|}} g(\cot (\theta / 2)) .
$$

Since the left side is greater or equal to 1 , as remarked above, we obtain (4).

For $V(x)=c \delta(x-b)$ the Birman-Schwinger operator reduces to the number $c\left(1-e^{-2 \sqrt{\mu} b}\right) /(2 \sqrt{\mu})$ and inequality (10) becomes equality provided $\sqrt{\mu} b$ satisfies $\left|1-e^{-2 \sqrt{\mu} b}\right|=g(\cot (\theta / 2))$. For given $m>0$ and $\theta \in(0,2 \pi)$ this determines $b$ and $|c|$. The phase of $c$ is found from the equation $c\left(1-e^{-2 \sqrt{\mu} b}\right) /(2 \sqrt{\mu})=1$.

Proof of Lemma 1.3. By continuity for $a>0$ there exists an optimizer $y_{0}$ such that $g(a)=\left|\mathrm{e}^{i a y_{0}}-\mathrm{e}^{-y_{0}}\right|$. We claim that $y_{0}$ satisfies $\pi / 3<a y_{0} \leq \pi$. To see the lower bound, note that $\left|\mathrm{e}^{i a y}-\mathrm{e}^{-y}\right| \geq 1$ if and only if $2 \cos (a y) \leq \mathrm{e}^{-y}$. In particular, $\cos \left(a y_{0}\right)<1 / 2$. For the upper bound, if $2 \pi>a y>\pi$ and $2 \cos (a y)<\mathrm{e}^{-y}$, replacing $y a$ by $2 \pi-y a$ leads to a contradiction. Similarly, if $y a>2 \pi$ it can replaced by $y a-2 \pi$ in order exclude that $y$ is the optimizer.

It is elementary to check that $\left|\mathrm{e}^{i a y}-\mathrm{e}^{-y}\right|$ is monotone increasing in $a$ for every fixed $y$ with $0 \leq y \leq \pi / a$. Since we know already that $y_{0} \leq \pi / a$, the monotonicity of $g$ follows.

Plugging in $y=\pi / a$, we obtain $g(a) \geq 1+\mathrm{e}^{-\pi / a} \geq 2-\pi / a$. For large enough $a$, it follows from this that $y_{0}$ is close to $\pi / a$. In particular, $y_{0} \geq \pi /(2 a)$, and hence $\left|\mathrm{e}^{i a y_{0}}-1\right| \geq g(a) \geq 2-\pi / a$. This implies that $y_{0}=\pi / a+O\left(a^{-2}\right)$, and thus $g(a)=2-\pi / a+O\left(a^{-2}\right)$, as claimed.

For an upper bound for small $a$, we use the triangle inequality and the bound $a y_{0} \geq \pi / 3$ to find $g(a) \leq 1+e^{-y_{0}} \leq 1+\mathrm{e}^{-\pi /(3 a)}$. 
Proof of Proposition 1.5. We proceed as in the proof of Theorem 1.1. The BirmanSchwinger operator has the kernel

$$
V(x)^{1 / 2} \frac{\mathrm{e}^{-\sqrt{\mu}|x-y|}+\frac{\sqrt{\mu}-\sigma}{\sqrt{\mu}+\sigma} \mathrm{e}^{-\sqrt{\mu}(x+y)}}{2 \sqrt{\mu}}|V(y)|^{1 / 2} .
$$

The assertion follows as above using that

$$
\sup _{y \geq 0}\left|1+\frac{\sqrt{\mu}-\sigma}{\sqrt{\mu}+\sigma} \mathrm{e}^{-2 \sqrt{\mu} y}\right| \leq 2
$$

by the triangle inequality and the fact that $|\sqrt{\mu}-\sigma| \leq|\sqrt{\mu}+\sigma|$. The fact that the bound (8) is sharp for given argument $0<\theta<2 \pi$ of the eigenvalue $\lambda$ follows by choosing $V(x)=-c i \mathrm{e}^{i \theta / 2} \delta(x)$ for $c>0$ and letting $c \rightarrow \infty$.

\section{REFERENCES}

[AAD] A. A. Abramov, A. Aslanyan, E. B. Davies, Bounds on complex eigenvalues and resonances. J. Phys. A 34 (2001), no. 1, 57-72.

[DN] E. B. Davies, J. Nath, Schrödinger operators with slowly decaying potentials. J. Comput. Appl. Math. 148 (2002), no. 1, 1-28.

[FLLS] R. L. Frank, A. Laptev, E. H. Lieb, R. Seiringer, Lieb-Thirring inequalities for Schrödinger operators with complex-valued potentials. Lett. Math. Phys. 77 (2006), no. 3, 309-316.

$[\mathrm{K}] \quad$ J. B. Keller, Lower bounds and isoperimetric inequalities for eigenvalues of the Schrödinger equation. J. Mathematical Phys. 2 (1961), 262-266.

[LS] A. Laptev, O. Safronov, Eigenvalue estimates for Schrödinger operators with complex potentials. Commun. Math. Phys. 292 (2009), no. 1, 29-54.

[LT] E. H. Lieb, W. Thirring, Inequalities for the moments of the eigenvalues of the Schrödinger Hamiltonian and their relation to Sobolev inequalities. In: Studies in Mathematical Physics, E. H. Lieb, et al. (eds.), 269-303. Princeton Univ. Press, Princeton, NJ, 1976.

[S] O. Safronov, Estimates for eigenvalues of the Schrödinger operator with a complex potential. Preprint arXiv:0902.3950v1 (2009).

Rupert L. Frank, Department of Mathematics, Princeton University, Princeton, NJ 08544, USA

E-mail address: rlfrank@math.princeton.edu

Ari Laptev, Department of Mathematics, Imperial College London, London SW7 2AZ, UK \& Department of Mathematics, Royal Institute of Technology, 10044 StockHOLM, SWEDEN

E-mail address: a.laptev@imperial.ac.uk \& laptev@math.kth.se

Robert Seiringer, Department of Physics, Princeton University, Princeton, NJ 08544, USA

E-mail address: rseiring@princeton.edu 